\title{
Xiao Yao San Improves Depressive-Like Behaviors in Rats with Chronic Immobilization Stress through Modulation of Locus Coeruleus-Norepinephrine System
}

\author{
Xiu-Fang Ding, ${ }^{1,2}$ Xiao-Hua Zhao, ${ }^{1}$ Yang Tao, ${ }^{3}$ Wei-Chao Zhong, ${ }^{1}$ Qin Fan, ${ }^{1}$ Jian-Xin Diao, \\ Yuan-Liang Liu, ${ }^{1}$ Yu-Yao Chen, ${ }^{1}$ Jia-Xu Chen, ${ }^{2}$ and Zhi-Ping $\mathbf{~ L v}^{1}$ \\ ${ }^{1}$ School of Traditional Chinese Medicine, Southern Medical University, Guangzhou, Guangdong 510515, China \\ ${ }^{2}$ School of Preclinical Medicine, Beijing University of Chinese Medicine, Beijing 100029, China \\ ${ }^{3}$ Comprehensive Department of Traditional Chinese Medicine of Shantou City Hospital, Shantou 515000, China
}

Correspondence should be addressed to Jia-Xu Chen; chenjiaxu@hotmail.com and Zhi-Ping Lv; lzping@fimmu.com

Received 19 September 2014; Accepted 25 November 2014; Published 25 December 2014

Academic Editor: Andreas Sandner-Kiesling

Copyright (c) 2014 Xiu-Fang Ding et al. This is an open access article distributed under the Creative Commons Attribution License, which permits unrestricted use, distribution, and reproduction in any medium, provided the original work is properly cited.

\begin{abstract}
Most research focuses on the hypothalamic-pituitary-adrenal (HPA) axis, hypothalamus-pituitary-thyroid (HPT) axis, and hypothalamus-pituitary-gonadal (HPGA) axis systems of abnormalities of emotions and behaviors induced by stress, while no studies of Chinese herbal medicine such as Xiao Yao San (XYS) on the mechanisms of locus coeruleus-norepinephrine (LC-NE) system have been reported. Therefore, experiments were carried out to observe mechanism of LC-NE system in response to chronic immobilization stress (CIS) and explore the antidepressant effect of XYS. Rat model was established by CIS. LC morphology in rat was conducted. The serum norepinephrine (NE) concentrations and NE biosynthesis such as tyrosine hydroxylase (TH), dopamine- $\beta$-hydroxylase (DBH), and corticotrophin-releasing-factor (CRF) in LC were determined. Results showed that there were no discernible alterations in LC in rats. The serum NE concentrations, positive neurons, mean optical density (MOD), and protein levels of TH, DBH, and CRF in model group were significantly increased compared to the control group. But XYS-treated group displayed a significantly decreased in NE levels and expressions of TH, DBH, and CRF compared to the model group. In conclusion, CIS can activate LC-NE system to release NE and then result in a significant decrease in rats. XYS treatment can effectively improve depressive-like behaviors in rats through inhibition of LC-NE neurons activity.
\end{abstract}

\section{Introduction}

Stress response is a risk factor that can develop affective or mental disorders, including anxiety, posttraumatic stress disorder, depression, and other disorders [1] and is characterized by the activation of the Locus coeruleus-norepinephrine (LC$\mathrm{NE}$ ) system. Indeed, LC-NE system can activate by multiple stressors, including social stress, footshock, novelty stress, and restraint. Furthermore, the effects of stress exposure can mimic the behavioral effects after activation LC, and damaged LC then attenuate neuroendocrine and behavioral responses $[2,3]$. The locus coeruleus in brainstem noradrenergic neurons during stress period can supply norepinephrine (NE) across the central nervous system (CNS) to form a LC-NE system and modulate the central stress response.
The NE contains axons that are widely distributed, indicating that this neurotransmitter plays a pivotal role in CNS function and behaviors $[4,5]$. The changes of behavioral depression found to be most closely related to stress were $\mathrm{NE}$ in the locus coeruleus. Norepinephrine-synthesizing enzymes including TH and DBH may be a neurochemical mechanism to interpret behavioral depression [6]. Both $\mathrm{TH}$ and $\mathrm{DBH}$ gene expressions in LC can be induced rapidly after immobilization stress $[6,7]$. Additionally, the role of activation of central CRF systems associated with mediation of behavioral responses to stressors. Summarized evidences show that stress can activate two forms of CRFnorepinephrine interactions. Either autonomic or emotional stress activates CRF release in the LC region, which in turn 
stimulates or activates LC-NE projection system [8, 9]. Administration of CRF antagonists within the LC can attenuate increased LC-NE neurotransmission induced by stressor $[10,11]$.

Many of studies have demonstrated that repeated or prolonged stressor can lead to complicated alterations in LC-NE neurotransmission. Although repeated or chronic stressors may not elicit NE to release, they can increase the capacity of this system to release NE, due to increased rates of NE synthesis [4]. At present, most research focuses on the hypothalamic-pituitary-adrenal (HPA) axis [12], hypothalamus-pituitary-thyroid (HPT) axis, and hypothalamus-pituitary-gonad (HPGA) axis systems of abnormalities of emotions and behaviors induced by stress $[13,14]$, while no studies of Chinese herbal medicine such as XYS or compounds on the mechanisms of LC-NE systems have been reported.

XYS is a Chinese herbal formula that was initially recorded in the book Taiping Huiming Heji Jufang in the Song Dynasty (960-1127 A.D.) in ancient China. The XYS finished products including decoction, powder, and pill have been widely used to treat mental disorders for thousands of years in China [15-17]. The therapeutic effects of XYS on ameliorating depressive-like behaviors or regulating expression of biochemical marker under stress period had been proved. For example, XYS can effectively regulate expression of tyrosine hydroxyls (TrkB), neurotrophin 3 (NT-3), neurotrophic factor (BDNF), leptin receptor (ob-R), and neuropeptide $\mathrm{Y}$ (NPY) in the hippocampal, arcuate nucleus and frontal cortex in rats with CIS $[18,19]$. XYS can inhibit IL-1 $\beta$ production in hippocampus and paraventricular nucleus (PVN) induced by repeated stress and alter metabolic network abnormalities in rats with chronic unpredictable mild stress (CUMS) or CIS [20-22]. In these investigations, the antidepressant effects of XYS on affective disorders were nearly all focused on hippocampus [21, 23], hypothalamus [24] (including arcuate nucleus [19]), and so on. While the research on Xiao Yao San regulates psychiatric affective disorders in mice locus coeruleus as an antidepressant medicine is lacking.

Towards that end, this study was designed to determine whether XYS can ameliorate depressive-like behaviors in rats with CIS through modulation of locus coeruleusnorepinephrine system based on our previous work [25].

\section{Materials and Methods}

2.1. Animals and Stress Procedure. The healthy Sprague Dawley male rats with bodyweight of 180-200 g were purchased from the Animal Center of Southern Medical University (number 2006-0015). All animals were fed in standard animal room (room temperature: $21 \pm 1^{\circ} \mathrm{C}$; relative humidity: $30 \%$ $40 \%$; light condition: a $12 \mathrm{~h}$ light-dark cycle). Food and purified water were available ad libitum. This experiment was approved by the Animal Ethics Committee of Southern Medical University and was performed strictly and conformed to the guidelines for the Care and Use of Laboratory Animals of China. All rats were housed individually and habituated for one week before performing further experiment. A total of 48 rats were arbitrarily assigned into four groups according to their weight. These comprised control group, model group, XYS treatment group, and fluoxetine treatment group. The treated groups were also subjected to CIS. The rats in the control group were fed routinely for twenty-one days; others were restrained by forcing them into an immobilizer device. In order to avoid the restraint adaptability of rats, CIS protocol made a minor modification according to previous studies $[21,26]$. In this experiment, immobilization time was randomly arranged and gradually increased on day 1 st for $1 \mathrm{~h}$ to $6 \mathrm{~h}$ on day 21st. Rats in control and model group were given $2 \mathrm{~mL}$ physiological saline by intragastric administration. Rats in XYS treatment group were given XYS decoction with the same ways according to their body surface area (dosage: $10 \mathrm{~g} / \mathrm{kg} / \mathrm{d}$ ). Rats in fluoxetine treatment group were given fluoxetine $(5 \mathrm{mg} / \mathrm{kg} / \mathrm{d})$. The physiological saline and drugs were intragastrically administered at 4:00 pm daily.

2.2. Preparation of Xiao Yao San. The herbal formula of XYS comprises the following drugs: Angelicae sinensis Radix (root of Angelica sinensis (Oliv.) Diels), Paeoniae Radix Alba (root of Paeonia lactiflora Pall.), Bupleuri Radix (root of Bupleurum chinese DC), Atractylodis Macrocephalae Rhizoma (root and rhizome of Atractylodes macrocephala Koidz), Glycyrrhizae Radix et Rhizoma (root and rhizome of Glycyrrhiza uralensis Fish), Poria (fungus nucleus of Poria cocos (Schw.) Wolf), Zingiberis Rhizoma Recens (root and rhizome of Zingiber officinale Rosc.), and Menthae Haplocalycis Herba (overground parts of Mentha haplocalyx Briq.) (the ratio is $6: 6: 6: 6: 6: 2: 2: 3)$. These herbal medicines were obtained from the Affiliated Nan Fang Hospital of Southern Medical University (Guangzhou, China) and authenticated by Liu Q, Department of Pharmacognostical Identification in School of Chinese Medicine of Southern Medical University. All herbal medicines were immersed in 10 times volume of distilled water and boiled at $80^{\circ} \mathrm{C}$ for $1 \mathrm{~h}$; the water extracts were then collected. This process was repeated again [21]. The extracts were concentrated by rotary Evaporator, and the final concentration was $1 \mathrm{~g} / \mathrm{mL}$. Fluoxetine (Lilly Suzhou Pharmaceutical Co., LTD, number J20080016) was obtained from the Affiliated Nan Fang Hospital of Southern Medical University and dissolved in distilled water. The final concentration was $0.2 \mathrm{mg} / \mathrm{mL}$. These solutions were stored at $-4^{\circ} \mathrm{C}$, and quality of XYS had been proved by high performance liquid chromatography-mass spectrometry analysis as reported in our previous research [23].

\subsection{Depressive-Like Behaviors and Body Weight in Rat with} CIS. Depressive-like behaviors in rat including action, diet, body weight, and defecation were observed during $21 \mathrm{~d}$ CIS period. The body weights of rats were measured on day 1st, day 7 th, day 14 th, and day 21 st. To examine the depressive behaviors in rats, a sucrose preference test (SPT) was measured on days 7 th, 14th, and 21st as previous described and made a minor modification [27]. Briefly, rats were singly habituated for $1 \mathrm{~d}$ prior to the test; their water bottles were replaced with two $50 \mathrm{~mL}$ bottles (one marked A: $1 \%$ sucrose solution; one marked B: water). The bottle positions were 

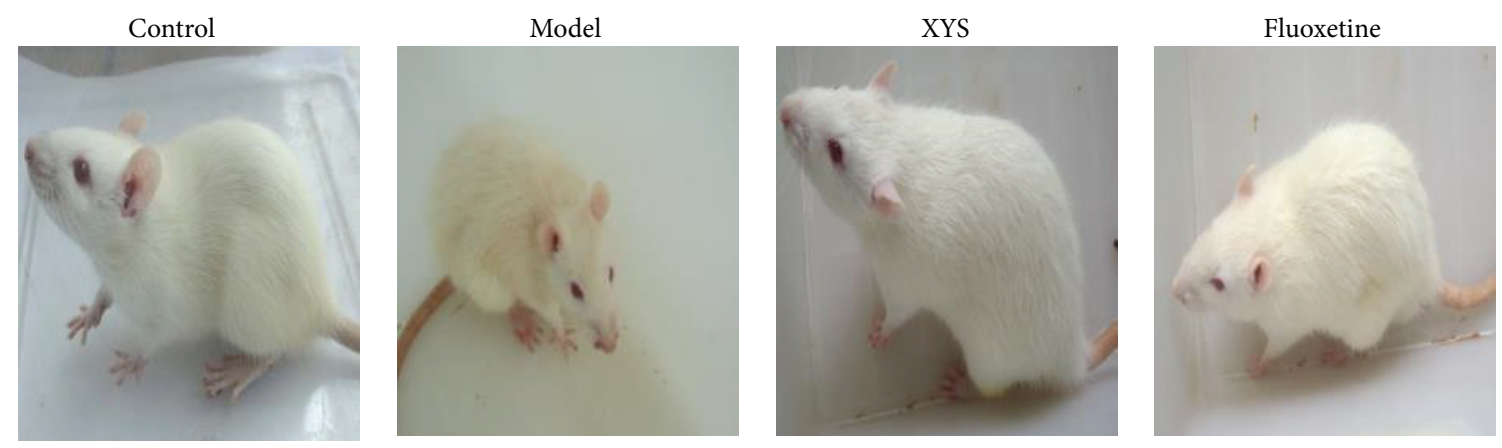

(a)

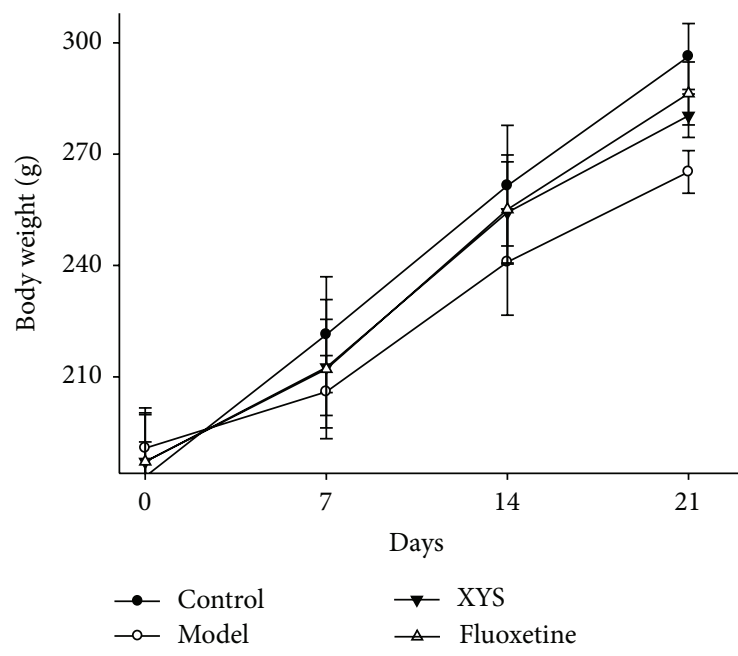

(b)

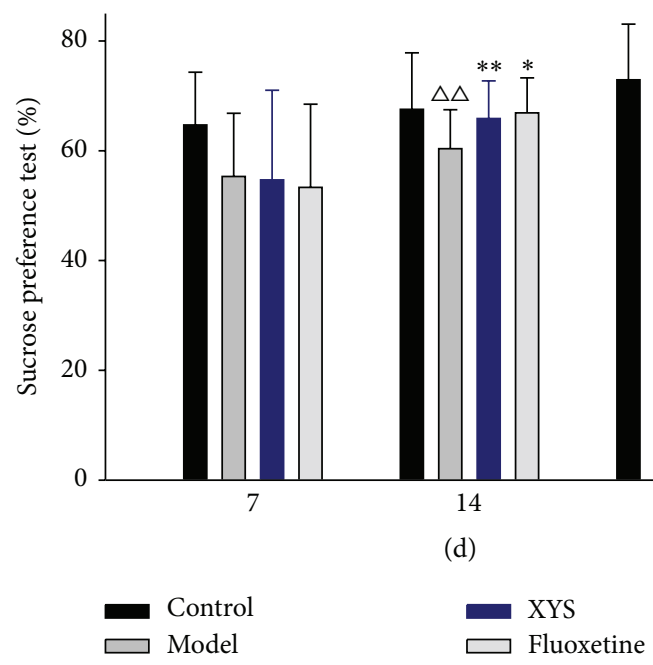

(c)

FIGURE 1: Effect of XYS on depressive-like behaviors of rats with CIS. (a) Actions and furs were observed in rats with CIS. (b) Rat body weight was recorded once a week during $21 \mathrm{~d}$-CIS period. (c) Sucrose preference test was conducted once a week during $21 \mathrm{~d}$-CIS period. Data were expressed as mean $\pm \mathrm{SD}, n=12$ per group. ${ }^{\triangle} P<0.01,{ }^{\triangle} P<0.05$ versus control, ${ }^{* *} P<0.01,{ }^{*} P<0.05$ versus model.

changed daily to prevent possible side bias. Rats were caged for $24 \mathrm{~h}$ to a $1 \%$ sucrose solution before measurement. This sucrose preference test was conducted weekly following water and food deprivation for $24 \mathrm{~h}$. The total fluid intake in two bottles was measured as VolA and VolB. The sucrose preference for each rat was calculated as $100 \times(\mathrm{VolA} /(\mathrm{VolA}+$ VolB)).

2.4. Noradrenaline (NE) Measurement. The experiment was terminated on the 21st day, and rats were anaesthetized with an intraperitoneal injection $10 \%$ chloral hydrate $(0.35$ to $0.40 \mathrm{~mL} / 100 \mathrm{~g}$ bodyweight). Venous blood was drawn from abdominal aorta and serum was then separated to measure NE levels. Enzyme-linked immunosorbent assay (ELISA) (CUSABIO, Wu Han, China) was measured using a microtiter plate reader (Victor3V, Perkin Elmer, Waltham, MA, USA) according to the protocol in the kit.

2.5. H\&E and Immunohistochemical Staining. Six animals in each group were anaesthetized with $2 \%$ pentobarbital sodium $(40 \mathrm{mg} / \mathrm{kg})$ and perfused with cold $0.9 \% \mathrm{NaCl}$ solution followed by perfusate solution (4\% paraformaldehyde, $2.5 \%$ glutaraldehyde, and $0.1 \mathrm{~mol}$ PBS). Brain stem contained locus coeruleus was cut out from brain tissues and fixed in 2.5\% paraformaldehyde solution for 48 hours and was then cut into serial sections $(5 \mu \mathrm{m})$. Preparation of tissue section and procedure of $\mathrm{H} \& \mathrm{E}$ staining were described in previous report [28].

For immunohistochemistry, the paraffin-embedded locus coeruleus sections were processed as free-floating slices, including deparaffinized, rehydrated, antigen retrieval, and then treated with hydrogen peroxidase in DDW (double distilled water) for $10 \mathrm{~min}$ at room temperature in order to inhibit endogenous peroxidase. Antigen retrieval was conducted by heating for $15 \mathrm{~min}$. Slices were incubated with primary antibodies (anti-TH, 1:1000 dilution; anti-DBH, 1:1000 dilution; anti-CRF, 1:500 dilution) after blocking in the antisera. After incubation with the secondary antibody, sections were placed in DAB reagent (ZSGB-BIO, Beijing, China) for 5-10 min at room temperature. After a further rinsing in PBS, sections were restained with hematoxylin and were mounted on gelatin-coated slides for observation under a light microscope. 
2.6. Western Blot Analysis. Protein levels of DBH, CRF, and $\mathrm{TH}$ were measured by Western blotting. The procedure was performed as previously described [23]. Briefly, proteins were extracted from locus coeruleus tissues, and concentrations were detected using a BCA protein assay kit (Beyotime, Shanghai, China). Based on the molecular weight of target protein, $12 \%$ separating gels were prepared, and concentration of stacking gels was $5 \%$. Protein gels were transferred to PVDF (polyvinylidene fluoride) membranes using a semidry blotter. For detection of the target protein, PVDF membranes were incubated in TBST with 5\% skimmed milk for $1 \mathrm{~h}$ at room temperature. After washing seven times for $5 \mathrm{~min}$ each in TBST, membranes were incubated first with primary antibodies for $2 \mathrm{~h}$ at room temperature or at $4^{\circ} \mathrm{C}$ overnight. Primary antibodies were anti-TH (1:2000 dilution), antiDBH (1:2000 dilution), anti-CRF (1:500 dilution), anti-betaactin (1:500 dilution), respectively.

After washing three times for $5 \mathrm{~min}$ each in TBST, membranes were incubated with horseradish peroxidase(HRP-) conjugated secondary antibody which was diluted in enclosed liquid $(1: 3000,1: 5000)$ for $1 \mathrm{~h}$ at room temperature. After three $5 \mathrm{~min}$ washes in TBST, membranes were developed using the enhanced chemiluminescence (ECL) detection reagent for $3 \mathrm{~min}$ and exposed to a CCD system (Image station $2000 \mathrm{MM}$, Kodak, Rochester, Rochester, NY, USA). The intensity of protein band was measured using an Image J software.

2.7. Statistical Analysis. All data were presented as the mean \pm SD and analyzed using an SPSS 17.0 statistical package. The multicomparison was performed. The mean values were conducted using one-way ANOVA as well as the homogenous variances test. The nonhomogeneous variances were compared by using Welch's test. $P<0.05$ was considered statistically significant difference.

\section{Results}

3.1. Behaviors and Body Weight in Rat with CIS. Rats were active and were in normal mood conditions with lustrous furs before modeling. In order to know the general status in rats under immobilization stress period, the variations of general behaviors in rats before and after modeling were observed. Rats in four groups were in an agitated state and prone to irritate and struggle during $7 \mathrm{~d}$ CIS period. After $10 \mathrm{~d}$ CIS, rats in model group showed passive manifestations and negative mood conditions. Rats in model group presented a fatigue, weak sounds, sluggish response, poor appetite, loose stool, and dull and yellow furs after stress for 14 days (Figure 1(a)). Body weights were significantly decreased on days 14 th and 21st after immobilization stress $(P<0.01)$. Body weights in rats were significantly increased after treating with XYS and fluoxetine (Figure 1(b)).

3.2. XYS Effect on 1\% Sucrose Preference in Rats with CIS. In order to know depressive behaviors in rats during immobilization stress period, $1 \%$ sucrose preference in rats was recorded on days 7 th, 14th, and 21st. A repeated measure

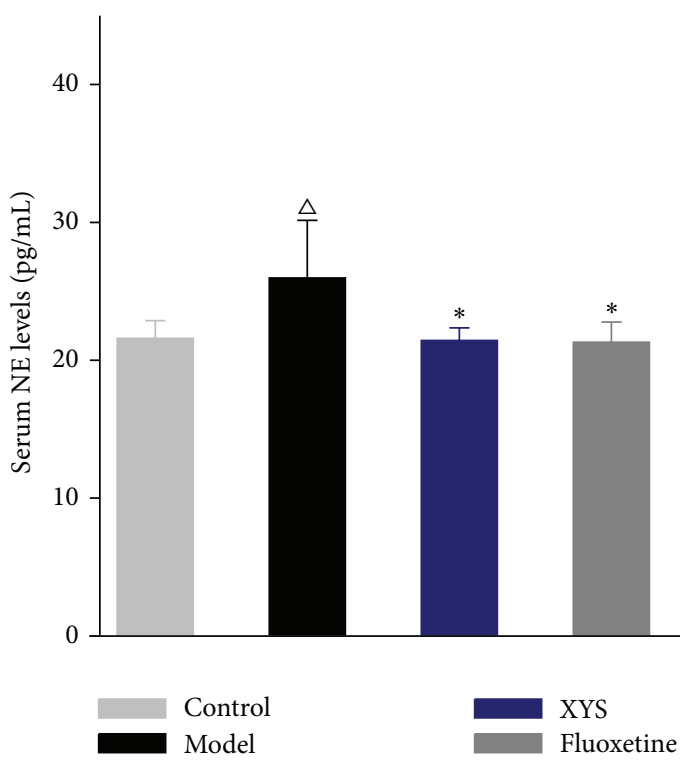

Figure 2: Effect of XYS on serum NE of rats with CIS. Data were expressed as mean $\pm \mathrm{SD}, n=12$ per group. ${ }^{\triangle} P<0.01$ versus control, ${ }^{*} P<0.01$ versus model.

ANOVA showed significant differences among four groups $(P<0.01)$. Three times measurements of sucrose preference in each group also showed significant differences $(P<0.01)$.

There was no interaction impact on time points and groups. No statistical difference was shown in SFT in rats subjected to CIS for 7 days. After modeling for two and three weeks, sucrose preference rate in rats in model group was lower than those in control group $(P<0.01, P<0.05$, resp.); compared with the model group, XYS treatment group significantly increased the sucrose preference $(P<0.01$, $P<0.05$, resp.). Fluoxetine treatment group also significantly increased the sucrose preference compared with the model group $(P<0.05)$ (Figure 1(c)).

3.3. XYS Effect on Serum NE Levels in Rats with CIS. In order to test whether chronic stress contributes to dysregulation of LC-NE system, peripheral NE concentrations were measured. Figure 2 indicated that serum NE levels were significantly different among four groups $(P<0.01)$. The serum levels of NE were $21.59 \pm 1.28,25.97 \pm 4.17,21.43 \pm 0.912$, and $21.31 \pm$ $1.46(\mathrm{ng} / \mathrm{mL})$ in the control, model, XYS, and fluoxetine treatment group, respectively. The statistics analysis showed that serum NE levels were significantly increased in the model group compared to the control group $(P<0.01)$. After XYS or fluoxetine treatment, NE levels were significantly decreased compared to the model group $(P<0.01)$.

3.4. LC Morphology and Effect of XYS on the Expressions of $T H, D B H$, and CRF in Rats with CIS. In order to evaluate the impact of CIS on gross LC morphology in rats, H\&E staining was conducted in this study. The results showed that LC adjacent located to the fourth ventricle in the brainstem 

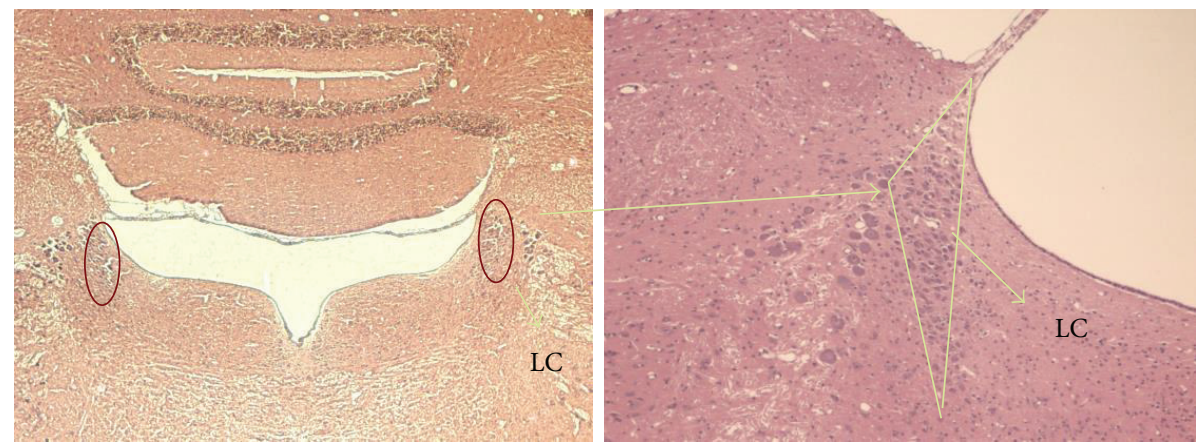

(a)
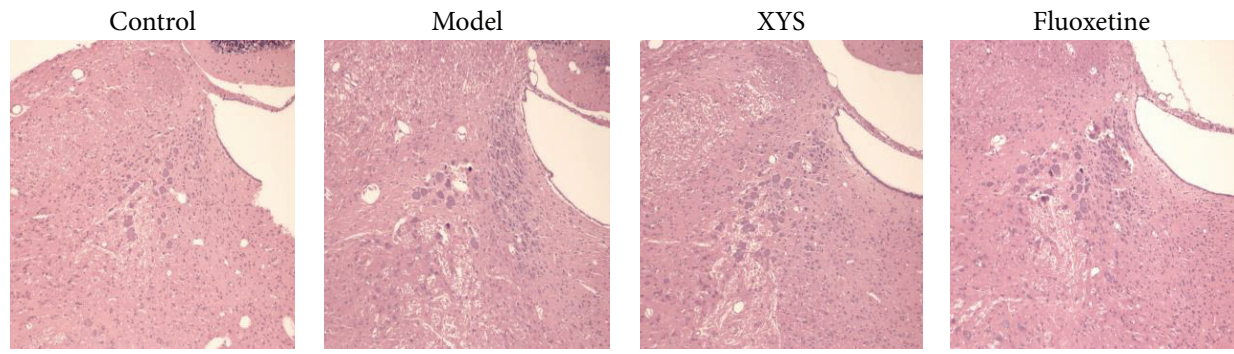

(b)

FIGURE 3: LC morphology in rats with CIS (H\&E staining). (a) Morphology and site of LC in rat. (b) Morphology of LC in rats after modeling for $21 \mathrm{~d}$ CIS among four groups. $n=6$ per group.

(Figure 3(a)). Furthermore, no discernible alterations were observed in LC structure in rats after exposure to CIS for 21 days (Figure 3(b)).

In order to determine whether CIS altered the function of LC neurons, TH, DBH, and CRF neurons were assessed by Immunohistochemistry. The results showed that LC expressed a number of $\mathrm{TH}, \mathrm{DBH}$, and CRF positive neurons in control group, $79.17 \pm 13.56,50.50 \pm 6.09$, and $46 \pm 9.40$, respectively. Quantity of the positive neurons of LC areas was increased and became dark brown, closely arranged, and round, shuttle or irregular shape of cell after modeling (Figures 4(a)-4(c)). In model group, the number of positive neurons of $\mathrm{TH}, \mathrm{DBH}$, and $\mathrm{CRF}$ was more than those of control group $(P=0.036,0.003$, and 0.002 , resp. $)$ and there was a significant difference. Similarly, MOD in model group was significantly enhanced compared to the control group ( $P=0.001,0.038$, and 0.007 , resp.). After treatment of XYS and fluoxetine, the number of positive neurons of TH, DBH, and CRF were significantly decreased compared with model group; likewise, MOD in these three neurons in model group was also significantly decreased compared to control group.

In order to examine protein expressions of $\mathrm{TH}, \mathrm{DBH}$, and CRF in rats were measured using Western blotting. Compared with the control group, protein levels of TH, CRF, and $\mathrm{DBH}$ in model group were significantly increased $(P=$ $0.002, P<0.01)$; protein levels of TH, CRF, and DBH in XYS and fluoxetine group were significantly decreased compared with the model group $(P=0.001,0.003, P<0.01)$. XYS and fluoxetine group had no obvious difference compared with the control group $(P>0.05$, Figure $4(\mathrm{~d}))$.

\section{Discussion}

Chronic immobilization stress is usually used as a model to assess the underlying alterations in cellular and molecular relevant to some depressive diseases [29-31]. Furthermore, antidepressant effect of XYS was approved by numerous literatures. Therefore, the present study used XYS to explore modulation of locus coeruleus-norepinephrine system in rats with CIS.

In order to adapt to various stress, a body can timely adjust physiological status. Moderate stress is beneficial to both body and mind, while excessive stress is disadvantage to a body's mental and physical health. This can be explained that rats adapted to CIS in the beginning in this study and were in an agitated state and prone to irritate and struggle after modeling for seven days. Rats could not adapt to this stress resulting in passive manifestations such as fatigue, sluggish, loose stool, body weight loss, and others after CIS for 14 days. This indicated that rats could not adjust themselves under excessive stress. After treating XYS that function is soothe liver and tonifying spleen, a trend to reverse the weight loss induced by stress was displayed.

Rodent is born with a preference for solutions or sweet foods. Decreased preference for sweet glucose solution in sucrose preference test means physical anhedonia, while therapies with chronic antidepressants can effectively reverse this reduction. SFT can detect the affective state or feelings of rodents [32]. To evaluate the depressive behaviors of rats with CIS in this experiment, a $1 \%$ sucrose preference protocol was used [27]; sucrose preference rate in first measurement 

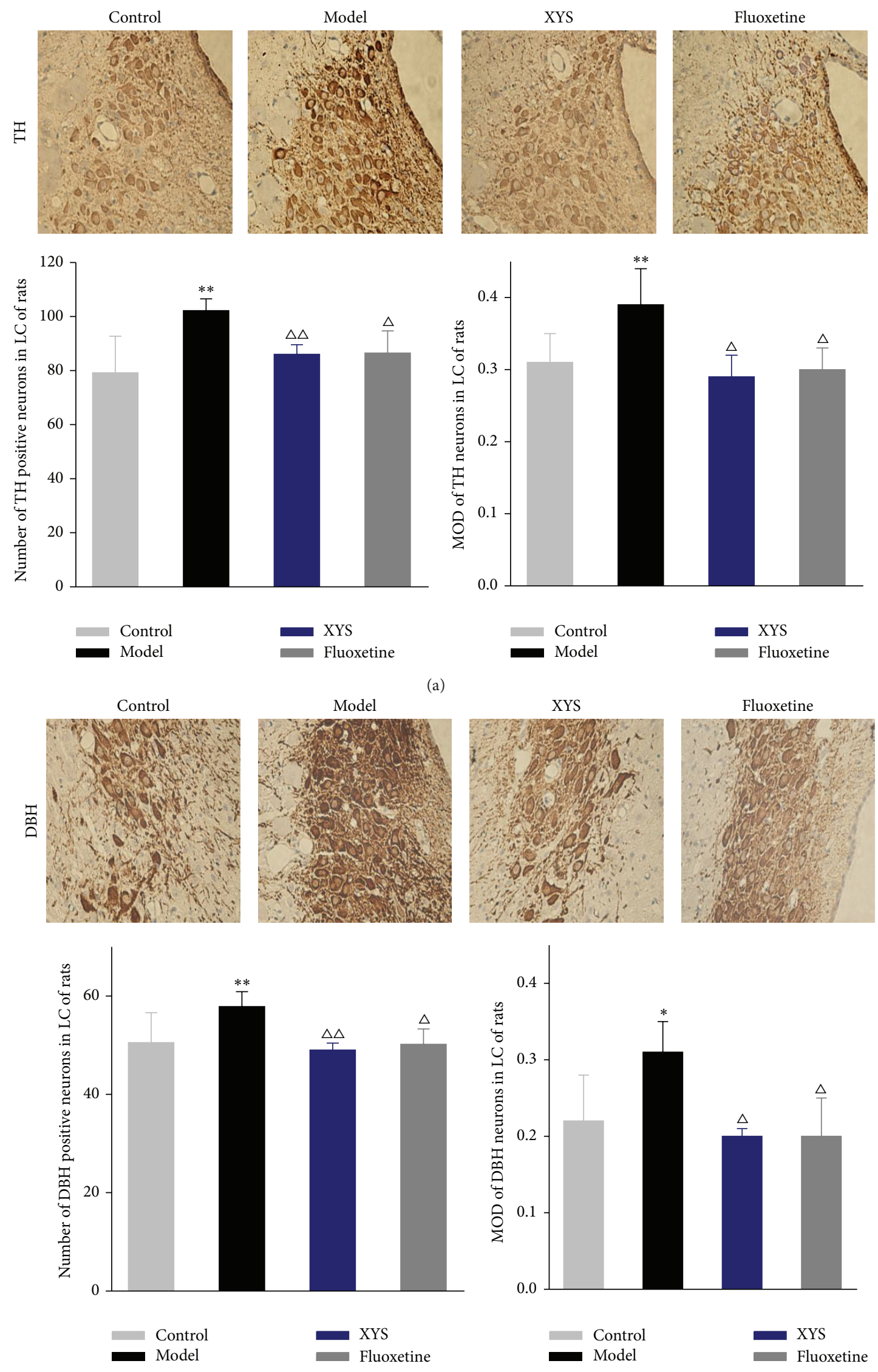

(b)

FIGURE 4: Continued. 

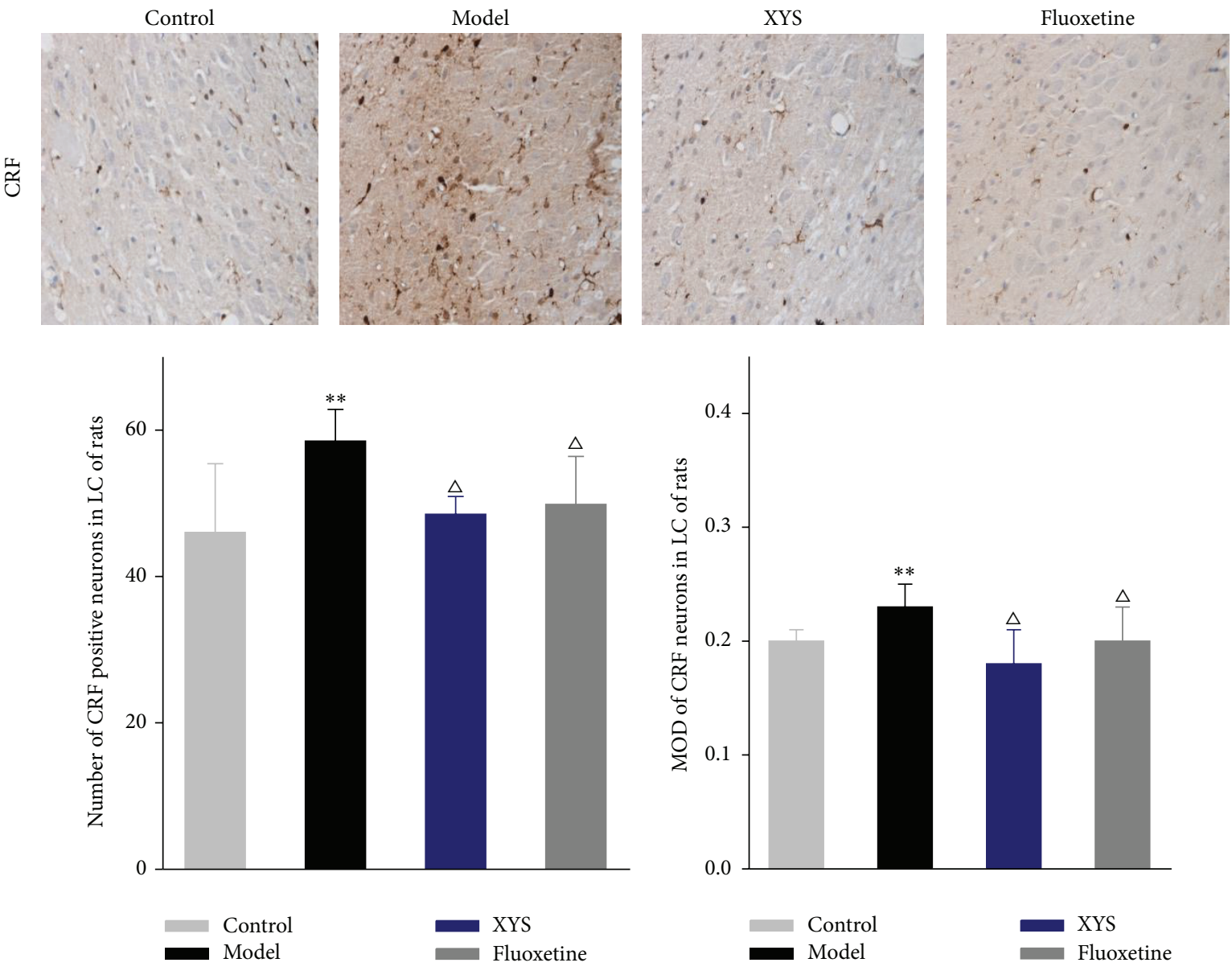

(c)
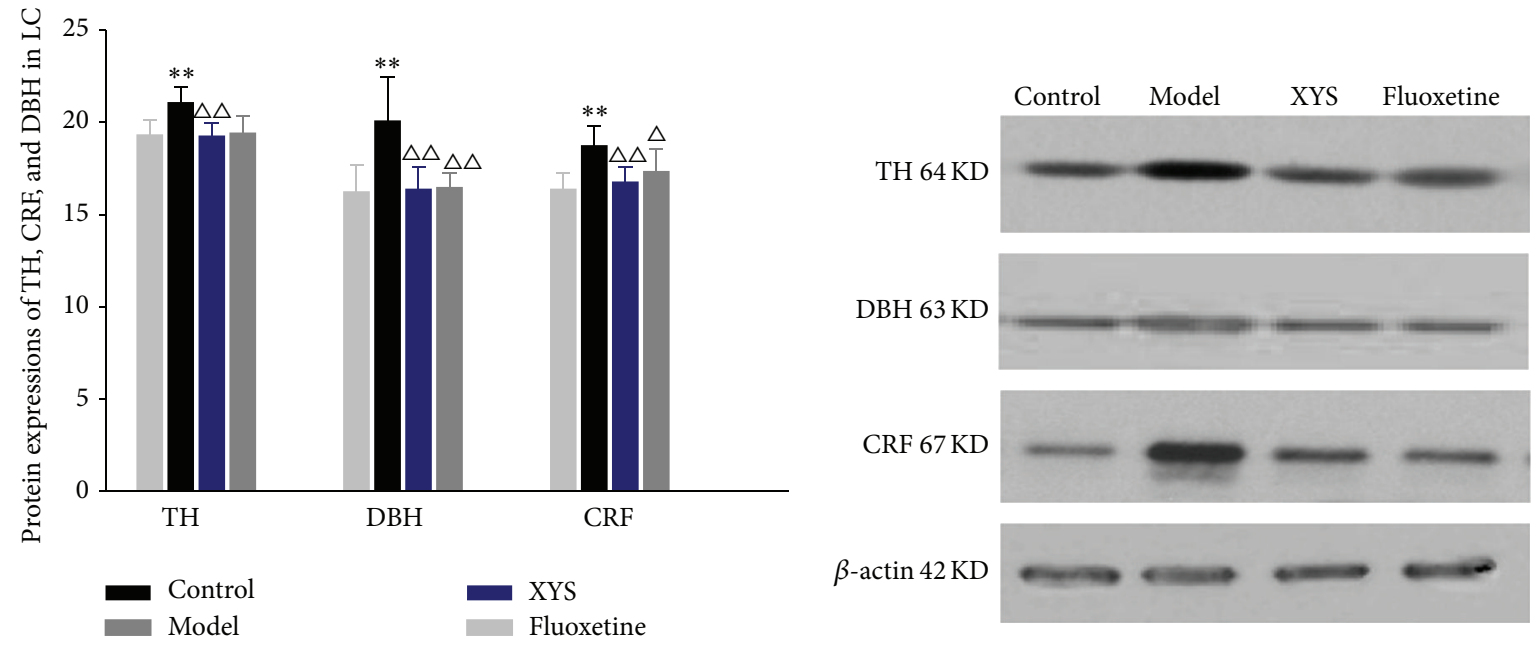

(d)

FIGURE 4: Effect of XYS on the expressions of TH, DBH, and CRF in LC of rats with CIS. ((a), (b), and (c)) The number of positive cells and mean density (SD) of TH, DBH, and CRF in LC of rats were determined by immunohistochemical staining. Data are expressed as mean \pm SD, $n=6$ per group. ${ }^{*} P<0.05,{ }^{* *} P<0.01$ versus control, ${ }^{\triangle} P<0.01$ versus model. (d) The protein expressions of TH, DBH and CRF in LC of rats were measured by Western blotting. Data were expressed as mean $\pm \mathrm{SD}, n=6$ per group. ${ }^{* *} P<0.01$ versus control, ${ }^{\triangle} P<0.05$, ${ }^{\triangle} \triangle P<0.01$ versus model. 
was lower than those on the second and third. The reason is that rats may have an adaptation process in the sugar consumption test. There was a relatively stable trend on the second and third measurement, but they were lower than the first measurement. This showed that rats could not adjust themselves under excessive physical stress. XYS treatment was effective in the amelioration of anhedonia induced by CIS as well as positive control.

LC-NE system firing can lead to differential NE levels to release. Specifically, NE system not only can be activated in response to acute stress across many preclinical studies, but also can be elicited by chronic stress paradigms. Varied outcomes in experiment studies depend on characteristics of stressor or individual. Findings from chronic psychosocial stress such as immobilization or cold restraint stressors have showed high NE reactivities and high plasma NE concentrations [33]. Thus, increased serum NE concentrations were observed in the model group. Chronic treatment with XYS caused a decrease in serum NE concentrations similar to that seen after treating fluoxetine. This result showed that XYS may ameliorate the depressive-like behaviors in rats with CIS through regulating NE concentrations.

Both $\mathrm{DBH}$ and $\mathrm{TH}$ were used to reveal the norepinephrine expression not only in locus coeruleus neurons, but also in the NE axons or fibers across the entire neuraxis $[34,35]$. Specifically, on the one hand, TH, the first enzyme in the norepinephrine biosynthesis, is widely utilized to identify the NE neurons [36]. On the other hand, $\mathrm{DBH}$, the final enzyme in norepinephrine biosynthesis, is commonly introduced to use a specific biomarker for NE fibers. CRF contains axon terminals and CRF binding sites; diverse behavioral effects elicited by intracerebral CRF support that it can serve as a modulator in neuronal activity to mediate behavioral or physiological effects in rodents $[37,38]$. Some substantial evidences also support that CRF has a neurotransmitter role in the locus coeruleus and moreover stated that CRF can be released into the locus coeruleus after locus coeruleusnorepinephrine system had been activated by various stressors and regulate locus coeruleus activation to facilitate behavioral or cognitive aspects of stress responses [39]. Therefore, in the present study, we analyzed the impact of immobilization stress on the expression of endogenous CRF and two genes involved in the biosynthesis of norepinephrine such as $\mathrm{TH}$ and $\mathrm{DBH}$.

In this study, no discernible changes in LC structure were found by $\mathrm{H} \& \mathrm{E}$ staining, suggesting that rats exposed to CIS for 21 days retained their brain structural integrity. Our results suggested increased positive neurons and MOD in $\mathrm{CRF}$ and synthesis enzyme of NE such as TH and DBH in the brain catecholaminergic system in response to 21-day chronic stress. It may contribute to a coordinated upregulation of these three genes expression. But these upregulation levels were decreased in response to XYS treatment as well as fluoxetine. We further confirmed these results in protein level by Western blotting in this experiment, and mRNA level had been confirmed in the former research [40]. Interestingly, our previous studies showed mRNA levels of $\mathrm{TH}$ and $\mathrm{DBH}$ were increased in response to CIS for $1 \mathrm{~h}$. These expressions were increased to the highest one after CIS for $3 \mathrm{~h}$ and then decreased after CIS for $6 \mathrm{~h}$ [25].

Taking these together, our studies indicate that LC-NE system can be activated by acute and chronic stress. But the activity of LC-NE system is rapid, transient in response to an acute stress. Contrarily, this activity is persistent in response to a chronic immobilization stress. We will further explore the mechanism of activation of the LC-NE system in response to chronic stress at different time points such as $7 \mathrm{~d}$ stress, $14 \mathrm{~d}$ stress, and other and identify differentially expressed proteins using a proteomics approach.

\section{Conclusion}

After exposure to an immobilization chronic stress, LC-NE system of rats can be activated resulting in a significant depression. Xiao Yao San can effectively improve depressivelike behaviors in rats. Further research is encouraged to detect the mechanism of activation of the LC-NE system in response to chronic stress in various time points and identify differentially expressed proteins or genes using an omics approach.

\section{Conflict of Interests}

The authors declare that there is no conflict of interests regarding the publication of this paper.

\section{Acknowledgments}

The authors thank Dr. Yukihiro Yamaguchi from the Department of Surgery of Johns Hopkins University and Dr. Lihong Shi from the Department of Pharmacology of Weifang Medical University for corrections of pharmacology part of this paper. This study was supported by grants from National Natural Sciences Foundation of China (nos. 81473597 and 81230085) and China Postdoctoral Science Foundation (no. 2014M550030).

\section{References}

[1] M. K. Green, C. S. S. Rani, A. Joshi et al., "Prenatal stress induces long term stress vulnerability, compromising stress response systems in the brain and impairing extinction of conditioned fear after adult stress," Neuroscience, vol. 192, pp. 438-451, 2011.

[2] W. J. Lipski and A. A. Grace, "Activation and inhibition of neurons in the hippocampal ventral subiculum by norepinephrine and locus coeruleus stimulation," Neuropsychopharmacology, vol. 38, no. 2, pp. 285-292, 2013.

[3] D. R. Ziegler, W. A. Cass, and J. P. Herman, "Excitatory influence of the locus coeruleus in hypothalamic-pituitaryadrenocortical axis responses to stress," Journal of Neuroendocrinology, vol. 11, no. 5, pp. 361-369, 1999.

[4] C. W. Berridge and B. D. Waterhouse, "The locus coeruleusnoradrenergic system: modulation of behavioral state and statedependent cognitive processes," Brain Research Reviews, vol. 42, no. 1 , pp. 33-84, 2003. 
[5] G. W. Dent, M. A. Smith, and S. Levine, "Stress-induced alterations in locus coeruleus gene expression during ontogeny," Developmental Brain Research, vol. 127, no. 1, pp. 23-30, 2001.

[6] D. L. Wong and A. W. Tank, "Stress-induced catecholaminergic function: transcriptional and post-transcriptional control," Stress, vol. 10, no. 2, pp. 121-130, 2007.

[7] B. Sun, X. Chen, L. Xu, C. Sterling, and A. W. Tank, "Chronic nicotine treatment leads to induction of tyrosine hydroxylase in locus ceruleus neurons: the role of transcriptional activation," Molecular Pharmacology, vol. 66, no. 4, pp. 1011-1021, 2004.

[8] G. F. Koob, "Corticotropin-releasing factor, norepinephrine, and stress," Biological Psychiatry, vol. 46, no. 9, pp. 1167-1180, 1999.

[9] D. M. Devilbiss, B. D. Waterhouse, C. W. Berridge, and R. Valentino, "Corticotropin-releasing factor acting at the locus coeruleus disrupts thalamic and cortical sensory-evoked responses," Neuropsychopharmacology, vol. 37, no. 9, pp. 20202030, 2012.

[10] M. E. Page, C. W. Berridge, S. L. Foote, and R. J. Valentino, "Corticotropin-releasing factor in the locus coeruleus mediates EEG activation associated with hypotensive stress," Neuroscience Letters, vol. 164, no. 1-2, pp. 81-84, 1993.

[11] R. J. Valentino, M. E. Page, and A. L. Curtis, "Activation of noradrenergic locus coeruleus neurons by hemodynamic stress is due to local release of corticotropin-releasing factor," Brain Research, vol. 555, no. 1, pp. 25-34, 1991.

[12] B. Gaire and D. Lim, "Antidepressant effects of Radix et Caulis Acanthopanacis Santicosi extracts on rat models with depression in terms of immobile behavior," Journal of Traditional Chinese Medicine, vol. 34, no. 3, pp. 317-323, 2014.

[13] Y. Y. Li, M. Xie, H. H. Wang, and Y. Chen, "Observation on changes of thyroid axis in rats with syndrome of liver depression and spleen deficiency and effect of Chaihu sijunzi decoction," Chinese Journal of Basic Medicine in Traditional Chinese Medicine, vol. 14, no. 3, pp. 191-195, 2008.

[14] Z. Xu, H. Zhang, S. Tan, X. Bian, and C. Fan, "Influence of acupoint catgut-embedding therapy on the hypothalamuspituitary-gonad axis in female chronic stress rats," Shanghai Journal of Acupuncture and Moxibustion, vol. 28, no. 1, pp. 5456, 2009.

[15] X. K. Qin, P. Li, M. Han, Z. J. Liu, and J. P. Liu, "Systematic review of randomize controlled trials of Xiaoyao powder in treatment of depression," Journal of Traditional Chinese Medicine, vol. 51, no. 6, pp. 500-505, 2010.

[16] Y. Zhang, M. Han, Z. Liu, J. Wang, Q. He, and J. Liu, "Chinese herbal formula Xiao Yao San for treatment of depression: a systematic review of randomized controlled trials," EvidenceBased Complementary and Alternative Medicine, vol. 2012, Article ID 931636, 13 pages, 2012.

[17] L. Bao, J. Chen, L. Huang et al., "Effects of Xiaoyao Wan on the behavioral despair and stress depression mice," Journal of Chinese Medicinal Materials, vol. 31, no. 9, pp. 1360-1364, 2008.

[18] J.-X. Chen, W. Li, X. Zhao, and J.-X. Yang, "Effects of the Chinese traditional prescription Xiaoyaosan decoction on chronic immobilization stress-induced changes in behavior and brain BDNF, TrkB, and NT-3 in rats," Cellular and Molecular Neurobiology, vol. 28, no. 5, pp. 745-755, 2008.

[19] S.-X. Wang, J.-X. Chen, G.-X. Yue, M.-H. Bai, M.-J. Kou, and Z.Y. Jin, "Xiaoyaosan decoction regulates changes in neuropeptide $y$ and leptin receptor in the rat arcuate nucleus after chronic immobilization stress," Evidence-Based Complementary and Alternative Medicine, vol. 2012, Article ID 381278, 16 pages, 2012.
[20] X. Gao, X. Zheng, Z. Li et al., "Metabonomic study on chronic unpredictable mild stress and intervention effects of Xiaoyaosan in rats using gas chromatography coupled with mass spectrometry," Journal of Ethnopharmacology, vol. 137, no. 1, pp. 690-699, 2011.

[21] H.-J. Park, H.-S. Shim, S. Y. Chung, T. H. Lee, and I. Shim, "Soyo-san reduces depressive-like behavior and proinflammatory cytokines in ovariectomized female rats," BMC Complementary and Alternative Medicine, vol. 14, article 34, 2014.

[22] Y. Dai, Z. Li, L. Xue et al., "Metabolomics study on the anti-depression effect of xiaoyaosan on rat model of chronic unpredictable mild stress," Journal of Ethnopharmacology, vol. 128 , no. 2, pp. 482-489, 2010.

[23] X. X. Zhu, O. D. Xia, W. H. Han et al., "Xiao Yao San improves depressive-like behavior in rats through modulation of $\beta$-arrestin 2-mediated pathways in Hippocampus," EvidenceBased Complementary and Alternative Medicine, vol. 2014, Article ID 902516, 13 pages, 2014.

[24] S. Wang, J. Chen, G. Yue, M. Bai, M. Kou, and Z. Jin, "Neuropeptide $\mathrm{Y}$ and leptin receptor expression in the hypothalamus of rats with chronic immobilization stress," Neural Regeneration Research, vol. 8, no. 18, pp. 1721-1726, 2013.

[25] Y. Tao, X. F. Ding, Y. Y. Chen et al., "Effects of acute stress on emotional behaviors and the expression of tyrosine hydroxylas and dopamine- $\beta$-hydroxylase in locus coeruleus of rats," Journal of Tropical Medicine, vol. 3, no. 11, pp. 274-277, 2011.

[26] L. Domínguez-Gerpe and M. Rey-Méndez, "Alterations induced by chronic stress in lymphocytes subsets of blood and primary and secondary immune organs of mice," $B M C$ Immunology, vol. 2, article 7, 2001.

[27] X. Cao, L.-P. Li, Q. Wang et al., "Astrocyte-derived ATP modulates depressive-like behaviors," Nature Medicine, vol. 19, no. 6, pp. 773-777, 2013.

[28] A. H. Fischer, K. A. Jacobson, J. Rose, and R. Zeller, "Hematoxylin and eosin staining of tissue and cell sections," Cold Spring Harbor Protocols, vol. 2008, no. 5, 2008.

[29] Y. Liang, X.-L. Guo, J.-X. Chen, and G.-X. Yue, "Effects of the Chinese traditional prescription Xiaoyaosan decoction on chronic immobilization stress-induced changes in behavior and ultrastructure in rat hippocampus," Evidence-Based Complementary and Alternative Medicine, vol. 2013, Article ID 984797, 8 pages, 2013.

[30] A. Wanasuntronwong, M. H. Tantisira, B. Tantisira, and H. Watanabe, "Anxiolytic effects of standardized extract of Centella asiatica (ECa 233) after chronic immobilization stress in mice," Journal of Ethnopharmacology, vol. 143, no. 2, pp. 579-585, 2012.

[31] A. Eşsizoǧlu, E. A. Yildirim, M. Mengi, T. Oral, and E. Yurdakoş, "The effects of 7-nitroindazole on anxiety and spatial memory in rats exposed to chronic immobilization stress," Nöropsikiyatri Arşivi, vol. 46, no. 4, pp. 157-162, 2009.

[32] “Animal models of depression," http://en.wikipedia.org/wiki/ Animal_models_of_depression.

[33] A. W. Goddard, S. G. Ball, J. Martinez et al., "Current perspectives of the roles of the central norepinephrine system in anxiety and depression," Depression and Anxiety, vol. 27, no. 4, pp. 339350, 2010.

[34] B. K. Hartman, D. Zide, and S. Udenfriend, "The use of dopamine $\beta$-hydroxylase as a marker for the central noradrenergic nervous system in rat brain," Proceedings of the National Academy of Sciences of the United States of America, vol. 69, no. 9, pp. 2722-2726, 1972. 
[35] T. Hokfelt, O. Johansson, K. Fuxe, M. Goldstein, and D. Park, "Immunohistoche-mical studies on the localization and distribution of monoamine neuron systems in the rat brain. I. Tyrosine hydroxylase in the mes -and diencephalon," Medical Biology, vol. 54, no. 6, pp. 427-453, 1976.

[36] J. Zhang, R. D. Darling, I. A. Paul et al., "Altered expression of tyrosine hydroxylase in the locus coeruleus noradrenergic system in citalopram neonatally exposed rats and monoamine oxidase A knock out mice," The Anatomical Record, vol. 294, no. 10, pp. 1685-1697, 2011.

[37] M. J. Owens and C. B. Nemeroff, "Physiology and pharmacology of corticotropin-releasing factor," Pharmacological Reviews, vol. 43, no. 4, pp. 425-473, 1991.

[38] D. T. Chalmers, T. W. Lovenberg, D. E. Grigoriadis, D. P. Behan, and E. B. de Souza, "Corticotrophin-releasing factor receptors: from molecular biology to drug design," Trends in Pharmacological Sciences, vol. 17, no. 4, pp. 166-172, 1996.

[39] R. J. Valentino and E. van Bockstaele, "Convergent regulation of locus coeruleus activity as an adaptive response to stress," European Journal of Pharmacology, vol. 583, no. 2-3, pp. 194203, 2008.

[40] X. F. Ding, Y. Tao, L. Chen et al., "Intervention effect of ease powder on locus coeruleus/noradrenergic system of rats with chronic unpredictable stress," Herald of Medicine, vol. 32, no. 11, pp. 1428-1431, 2013. 


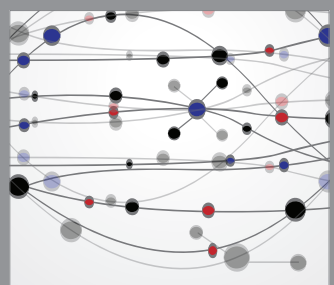

The Scientific World Journal
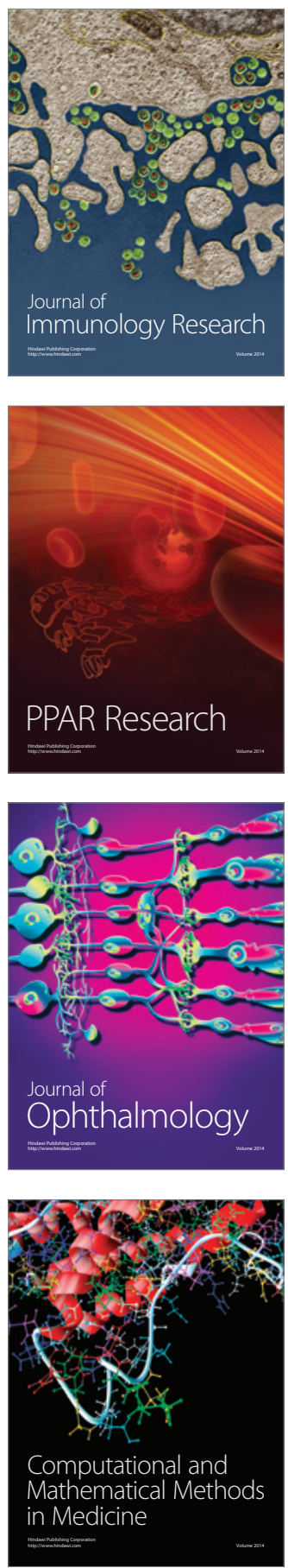

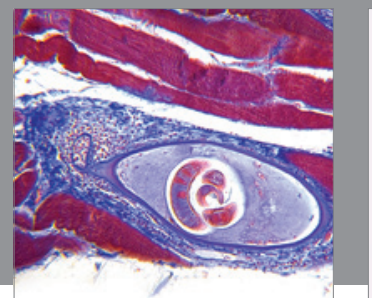

Gastroenterology

Research and Practice
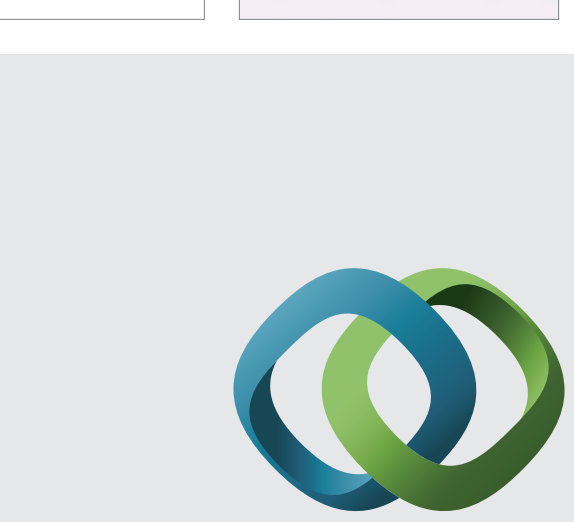

\section{Hindawi}

Submit your manuscripts at

http://www.hindawi.com
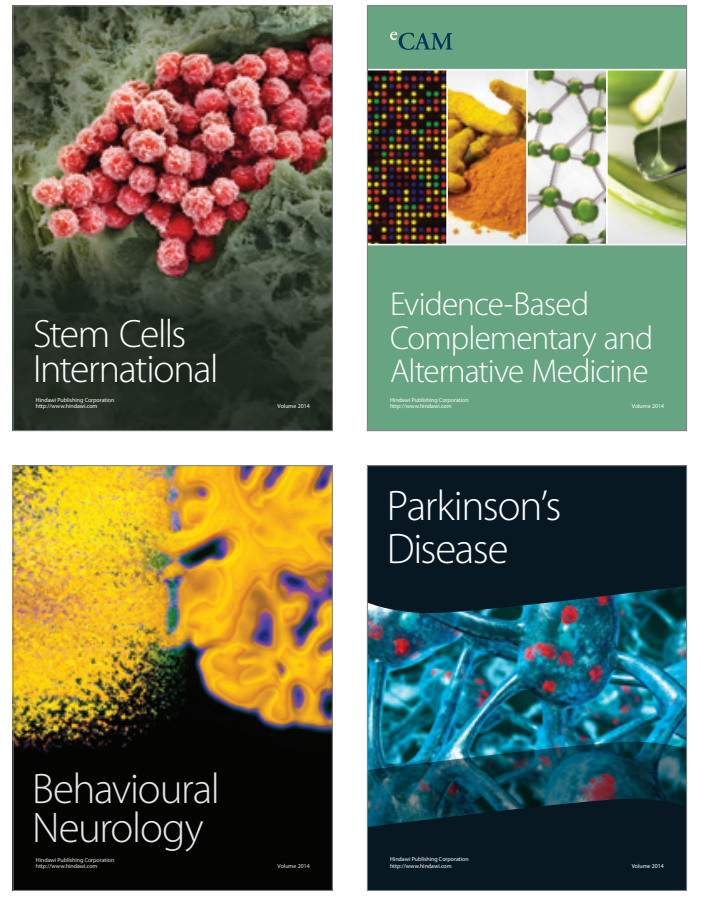
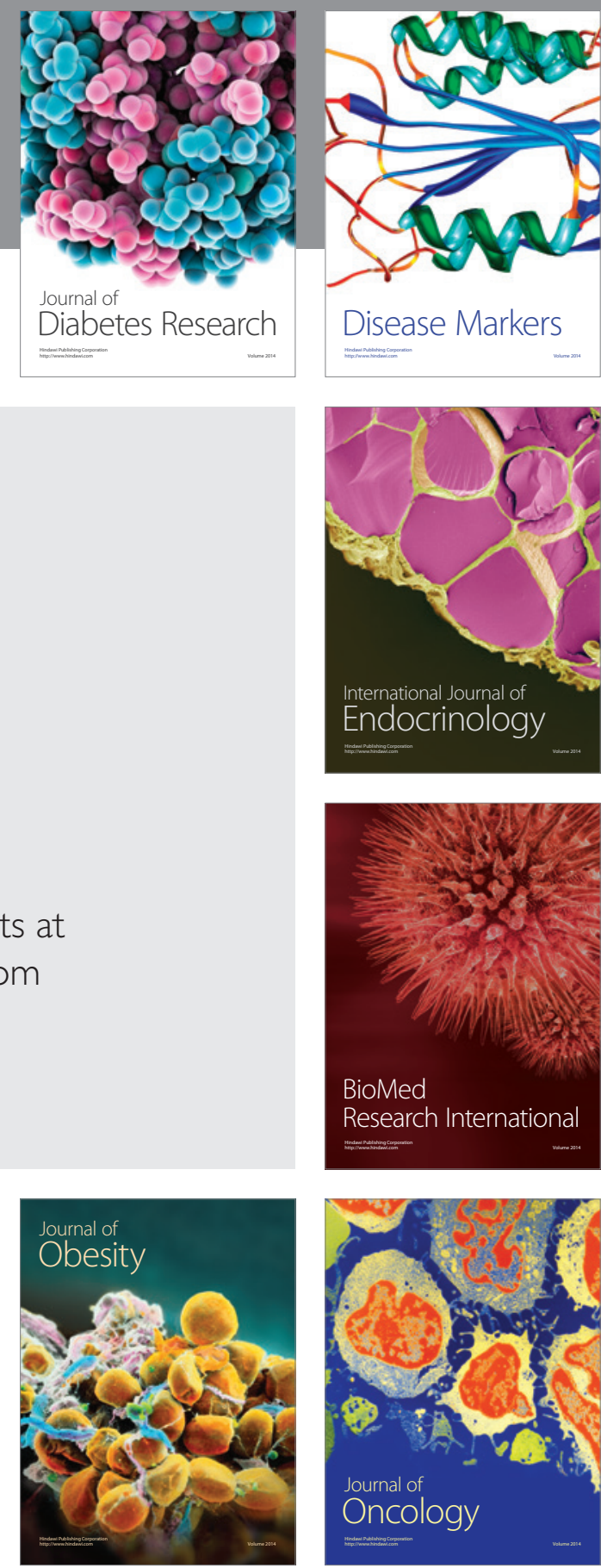

Disease Markers
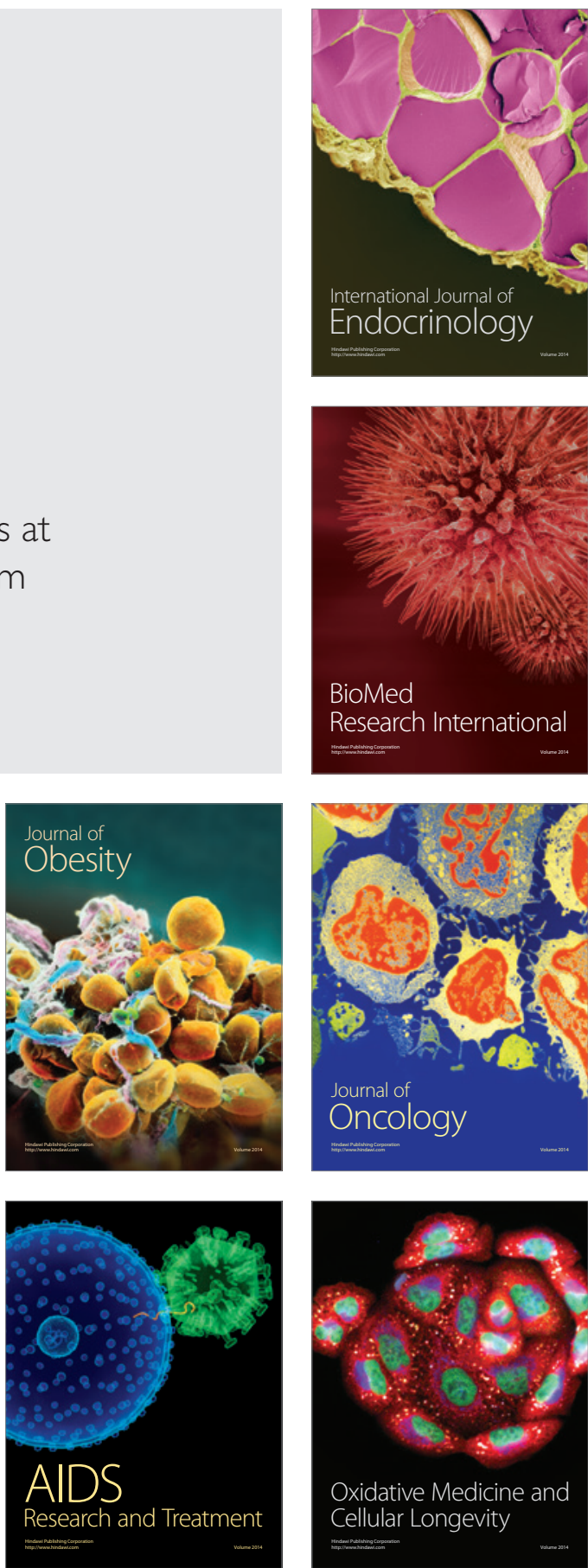